CASO CLÍNICO

\title{
Reto diagnóstico: Hipoacusia, Enteropatía Autoinmune y Linfadenopatia de Kikuchi - un debut inusual de LES
}

\section{[Diagnostic challenge: hearing loss, autoimmune enteropathy and Kikuchi lymphadenopathy - An unusual debut of SLE]}

\author{
Dra. Ana Karina Coronado', Dr. Ricardo McCalla ${ }^{2}$, Dra. Dorothee Stichweh ${ }^{3}$, Dra. Geneva Rodríguez ${ }^{4}$ \\ 'Pediatra. Gastroenteróloga. Hospital Materno Infantil José Domingo de Obaldía. ?Pediatra Gastroenterólogo. Hospital del Niño Dr. José Renán Esquivel, \\ Panamá. ${ }^{3}$ Pediatra Reumatóloga. Hospital del Niño Dr. José Renán Esquivel, Panamá. ${ }^{4}$ Patóloga. Hospital del Niño Dr. José Renán Esquivel, Panamá \\ Correspondencia:Dra.Ana Karina Coronado. / Correo electrónico:karina_coronado@hotmail.com
}

Recibido: 12 de noviembre de 2020

Aprobado: 22 de marzo de 2021

Publicado: 24 de junio de 2021.

DOI: $10.37980 /$ im.journal.rspp.20211634

Cita: Coronado AK, et. al, (2020), Hipoacusia, Enteropatía Autoinmune y Linfadenopatia de Kikuchi - un Debut Inusual de LES, DOI:

Palabras claves: síndrome febril, enteropatía autoinmune, lupus eritematoso sistémico, linfadenitis necrotizante histiocítica,.

Keywords: febrile syndrome, autoimmune enteropathy, systemic lupus erythematosus, histiocytic necrotizing lymphadenitis.

Reproducción: Artículo de acceso libre para uso personal e individual. Sujeto a derechos de reproducción para otros usos.

Conflictos de interés: Los autores declaran no tener conflictos de interés.

Financiamiento: El autor no declara fuentes externas de financiamiento asociados a este trabajo.

\section{Resumen}

El lupus eritematoso sistémico (LES) puede presentarse de muchas maneras y puede ser difícil de diagnosticar. En ocasiones puede ser difícil distinguir la enfermedad subyacente, ya que se sabe que el LES mismo causa síntomas gastrointestinales e incluso malabsorción. Este caso refleja los desafíos de diagnóstico que enfrentamos durante la larga y complicada hospitalización de una adolescente con compromiso sistémico, síndrome febril, malabsorción, marcadores inflamatorios elevados, hipoacusia, nefritis, linfadenitis necrotizante histiocítica.

\section{Abstract}

Systemic lupus erythematosus (SLE) can present in many ways and can be difficult to diagnose. It can sometimes be difficult to distinguish the underlying disease, as SLE itself is known to cause gastrointestinal symptoms and even malabsorption. This case reflects the diagnostic challenges we faced during the long and complicated hospitalization of an adolescent with systemic compromise, febrile syndrome, malabsorption, elevated inflammatory markers, hearing loss, nephritis, histiocytic necrotizing lymphadenitis.

\section{Reporte de caso}

Femenina de 13 años con antecedente de intolerancia a la lactosa y estreñimiento. Sin antecedentes familiares patológicos. Cuadro de hipoacusia bilateral súbita, de 6 meses de evolución previo a su ingreso.

Ingresa por cuadro de 3 semanas de evolución de fiebre persistente sin predominio horario asociado a evacuaciones líquidas, sin moco ni sangre, dolor abdominal y pérdida de peso. Tratada ambulatoriamente con antibióticos.

Al examen físico, estado nutricional con bajo peso para su edad. Mucosa oral sin lesiones. Cuello con adenopatías cervicales anteriores, la de mayor tamaño de $1.5 \mathrm{~cm}$ cervical izquierdo móvil no dolorosa. Adenopatías submandibulares e inguinales pequeñas. Piel sin lesiones. Abdomen blando depresible, sin visceromegalia palpable. Piel sin exantemas
Extremidades simétricas sin edema, sin artritis, con cianosis ungueal.

Oídos con Hipoacusia profunda bilateral (sin respuesta a máximos intensidades con tono agudo). Timpanometría adecuada movilidad de las membranas timpánicas con adecuada presión de oído medio. Reflejos estapediales ausentes ipsi y contralaterales, asociado con pérdida auditiva bilateral. Curva de hipoacusia profunda con resto auditivos en ambos oídos y sin disociación fonémica. Inadecuada respuesta de intermodulación bilateral compatible con pérdida auditiva en ambos oídos.

Estudios de laboratorio con anemia normocítica normocrómica 8.3g/dL; marcadores inflamatorios positivos (VES 65 $\mathrm{mm} / \mathrm{hr}$ ), enzimas hepáticas con elevación leve (TOA : $49 \mathrm{U} / \mathrm{L}$ TGP : $57 \mathrm{U} / \mathrm{L})$ 
Los resultados de exámenes de laboratorio practicados como parte del síndrome febril demostraron: heces por Rotavirus, Clostridium, Campylobacter, Norovirus negativos; CMV, EBV, parvovirus B19 negativos; PPD negativa. Histoplasmosis, Bartonella Henselae, malaria, HIV negativo . Herpes virus tipo 7 positivo. Uroanálisis iniciales con énfasis en proteinuria negativa, sangre oculta negativa.

Biopsia de ganglio cervical reporta linfadenitis necrotizante histiocítica, tinciones de inmunohistoquímica positiva para células de interés: Mieloperoxidasa, CD68, CD8, CD20.

Tomografía toracoabdominal reporta hepatoesplenomegalia difusa. Ganglios linfáticos reactivos axilares e inguinales bilaterales, adenopatías paraaórticas.

Ultrasonido abdominal reporta hepatoesplenomegalia y patrón micronodular del bazo. Hallazgos inespecíficos que pudiera relacionarse con patología infecciosa vs infiltrativa.

A nivel gastrointestinal pANCA y calprotectina negativas. Transglutaminasa IgA positivo a título bajo(11.7), IgG negativa (8.5). Antiendomisio positivo. Enterotomografía sin imágenes patológicas. Estudio molecular para enfermedad celiaca reporta fenotipo portador DQ2 trans (Hp1) + DQ2cis. Biopsia intestinal con hallazgos histopatológicos de atrofia de vellosidades y linfocitos intraepiteliales.

Hallazgos histopatológicos de endoscopia alta-colonoscopía: Gastritis crónica folicular moderada sin actividad. Negativo por Helicobacter pylori. Duodeno: Ver figura 2. Colon al azar: colitis microscópica linfocítica. Recto con arquitectura histológica conservada.

Durante la 4ta semana de hospitalización cursa con compromiso sistémico, persiste febril desde su ingreso a pesar de cobertura antibiótica de amplio espectro, con pancitopenia (descenso de hemoglobina $7.5 \mathrm{~g} / \mathrm{dL}$, neutropenia, trombocitopenia), hipoalbuminemia. Persiste con velocidad de eritrosedimentación elevada. Durante el curso de la enfermedad a nivel renal cursa con datos de nefritis (hematuria macroscópica y proteinuria). Se realiza biopsia renal: glomerulonefritis proliferativa mesangial difusa con depósitos mesangiales y arteriolares de C3. Nefropatía mesangial C3. Por el comportamiento clínico inicialmente consideramos patrón autoinmune asociado.

Pruebas de autoinmunidad anticuerpos antinucleares (ANA), antiDNA, factor reumatoideo inicialmente resultaron negativos, fracción de C3 bajo (C3: 50.6 N: 125 (83-177); C4: 16 N: 28 (15-45). Otras pruebas inmunológicas demuestran elevación de $\lg \mathrm{E}, \operatorname{lgG}$ ( IgE: >1000 Ul/mL rango normal 23.6 (1.5-114) ; IgG: 3509.5 mg/dL rango normal 994 (639-1349) ; IgM: 113.4 rango normal (56-362), lgA: $258.7 \mathrm{mg} / \mathrm{dl}$ (70-317) en rango normal.
Figura 1 Cianosis ungueal

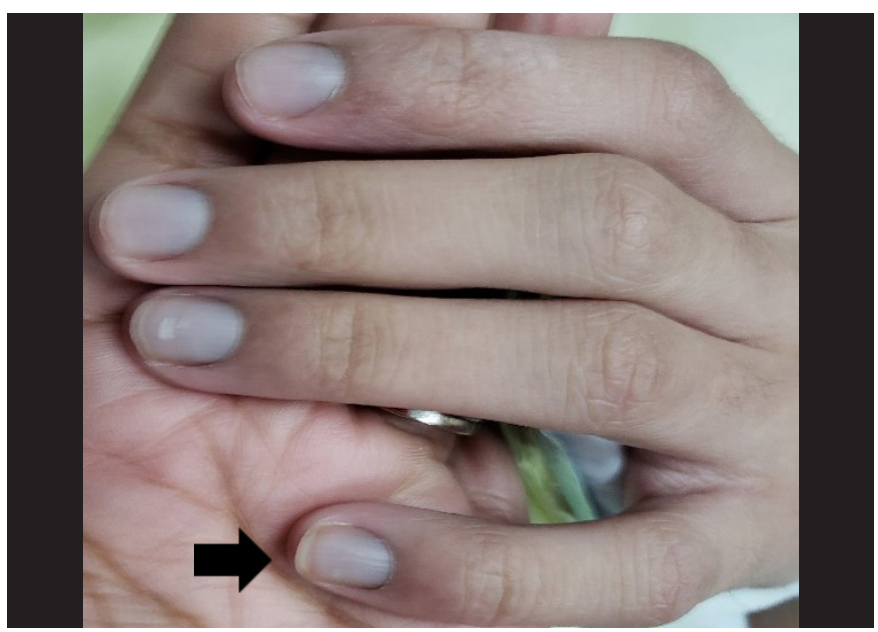

Figura 2: A y B: Hallazgos endoscópicos: aspecto festoneado de la mucosa duodenal. C y D: Duodeno: vellosidades acortadas y aumento significativo de linfocitos intraepiteliales, 20 a 30 LIE por 100 enterocitos (Marsh IIIb). Hiperplasia de las criptas.
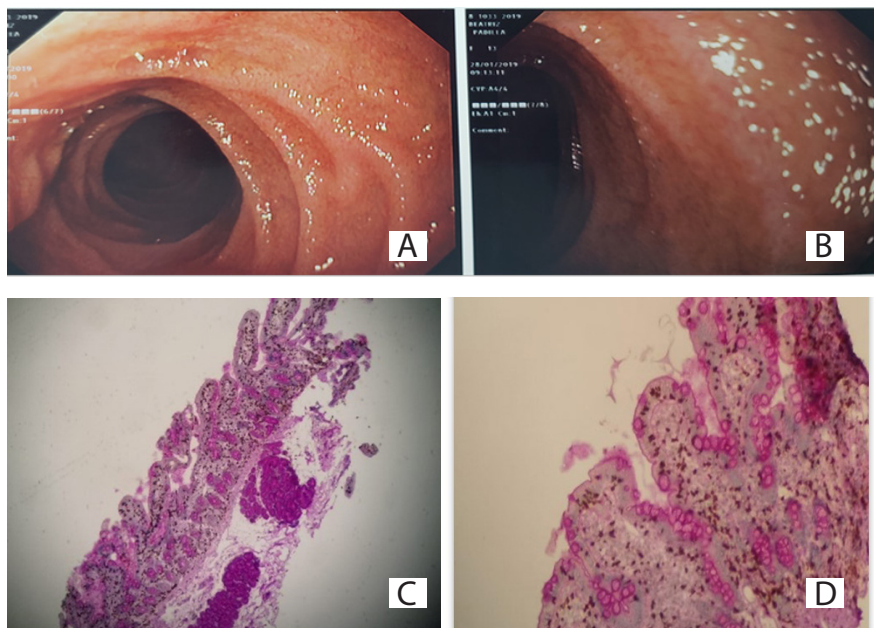

En este caso se realizó el diagnóstico diferencial con causas infecciosas, hematológicas (médula ósea con trama medular positiva, neutrofilia, no células infiltrativas ni parásitos, mielocultivo negativos). Inmunológico con disminución de subpoblaciones de linfocitos.

Con lo anterior y la evolución desfavorable de la paciente se decide iniciar pulsos de metilprednisolona y dieta sin gluten, suplementación con vitamina B12, hierro y ácido fólico, con mejoría de la sintomatología. Los síntomas mejoran paulatinamente incluyendo la hipoacusia, mejoría del gasto fecal, sin presentación de nuevos picos febriles. Posteriormente se mantiene con prednisona vía oral a $2 \mathrm{mg} / \mathrm{kg} /$ día. Un año después, luego de la disminución progresiva, se omiten corticoides con reagudización de síntomas a la semana presentando artralgias, mialgias y fiebre. Se realizan nuevamente pruebas de autoinmunidad resultando Anti RO, anti LA, anti SM, anti 
RNP negativo, el Anti-dsDNA positivo (191.8 IU/mL), ANA Hep2 positivo 1/320, C3 disminuido (66.7mg/dL), C4 disminuido $(11.6 \mathrm{mg} / \mathrm{dL})$ con lo que finalmente se integra el diagnóstico de Lupus eritematoso sistémico, con manifestación gastrointestinal (enteropatía autoinmune) y Linfadenitis de Kikuchi Fujimoto e hipoacusia neurosensorial bilateral.

\section{Discusión}

El lupus eritematoso sistémico (LES) puede afectar prácticamente todos los órganos y sistemas del cuerpo. ${ }^{1}$ Los síntomas gastrointestinales (GI) son comunes en pacientes con LES, ocurriendo hasta en un $40 \%$. Los síntomas suelen ser leves y la mayoría son causados por infecciones virales o bacterianas o reacciones adversas a la terapia. Aunque la afectación GI relacionada con el LES no es tan común como en la piel, articulaciones o renal, diagnóstico y tratamiento precoces y precisos son fundamentales para mejorar el pronóstico. ${ }^{\top}$

Consideramos la realización de pruebas para descartar enfermedad celiaca (EC), enfermedad inflamatoria intestinal en este caso por datos de: bajo peso severo, diarrea persistente, distensión abdominal, estreñimiento, anemia ferropénica, VES elevados, aunado la sospecha de presencia de otras enfermedades autoinmunes por su compromiso sistémico.

Se sospecho asociación del cuadro con una EC en crisis por los datos de diarrea crónica, malabsorción y hallazgos histopatológicos compatibles con atrofia de vellosidades (Marsh III), presencia de HLA DQ2, sin embargo con prueba para transglutaminasa positivo a títulos bajos. Cabe destacar que alrededor del $30 \%$ de la población general también es HLA DQ2 positivo. No obstante, es necesario aclarar que la EC es una enfermedad multigenética $y$, por lo tanto, la expresión de HLA-DQ2/HLA-DQ8 es una condición necesaria pero no suficiente para el desarrollo de la enfermedad.

Los títulos elevados de AATG IgA, superiores a 10 veces el LSN, predicen la existencia de lesión de las vellosidades intestinales con gran especificidad. En algunas ocasiones se han descrito valores falsos positivos, habitualmente a títulos bajos, por reacción cruzada con anticuerpos en otras situaciones clínicas, como enfermedades autoinmunes, enfermedades hepáticas e infecciones. ${ }^{2,3,4}$

Por otro lado, la enteropatía autoinmune (EAI) debe ser considerada en el diagnóstico diferencial de malabsorción con atrofia de las vellosidades del intestino delgado. Aunque la EAl es más común en pacientes pediátricas, esta enfermedad rara vez se observa en adultos y suele asociado con una predisposición a trastornos autoinmunes. El diagnóstico de esta condición se basa en cinco criterios: diarrea crónica durante más de 6 semanas, malabsorción, hallazgos histológicos específicos, exclusión de trastornos similares y la presencia de anticuerpos específicos como anti-enterocitos. ${ }^{1}$
La enteropatía lúpica puede presentarse como una enteritis isquémica aguda o una enteropatía perdedora de proteínas. El dolor y la diarrea pueden reflejar enteritis o pueden desarrollarse como consecuencia de una enfermedad mesentérica de LES, vasculitis o trombosis.

Otras causas de atrofia vellositaria descritas lo son : Giardiasis, inmunodeficiencia común variable, tuberculosis intestinal, linfoma intestinal, sprue tropical, enfermedad de Whipple, entre otras.

La evaluación histológica de la EAl demuestra cambios en las vellosidades del intestino delgado que incluyen atrofia, generalmente más prominentes en el intestino proximal. Los abscesos de las criptas también pueden ser visto. Puede haber cuerpos apoptóticos y linfocíticos. Infiltración en el epitelio de la cripta con relativa escasez de linfocitosis de superficie ( $<40$ linfocitos por 100 epitelio células), mientras que una distribución intraepitelial de linfocitos predomina en la enfermedad celíaca. ${ }^{5}$

Una de las manifestaciones iniciales en este caso fue la hipoacusia, seguido de un síndrome febril, los cuales nos hicieron sospechar proceso autoinmune asociado, aunado las manifestaciones gastrointestinales. La hipoacusia neurosensorial (HNS) autoinmune es una afección del oído interno de etiología desconocida, con una patogenia autoinmune. La relación del LES con la HNS autoinmune, es poco conocida; se han publicado casos aislados o escaso estudios de enfermos con LES con un número muy limitado de casos. Las afectaciones o lesiones de mayor incidencia en el órgano auditivo descritas son: vértigo/mareo, tinnitus/acufenos, lesiones cocleares (estría vascular, ligamento espiral y en el interior de arteria auditiva) e inflamación vascular (vasculitis). 5,6,7 En nuestro caso, la hipoacusia mejoró luego de inicio de terapia con corticoides.

Lupus eritematoso sistémico (LES) es un trastorno multisistémico con manifestaciones que incluyen erupción cutánea, artritis, citopenia y enfermedad renal. ${ }^{8}$ En este caso inicialmente solo se presentó citopenias y datos de nefritis. Se observan anemia, trombocitopenia y leucopenia en 50 a $75 \%$ de pacientes. La anemia más común es la anemia normocítica normocrómica, que, cuando persiste, suele convertirse en anemia microcítica e hipocrómica. ${ }^{9}$

La esplenomegalia, que ocurre en el $20 \%$ al $30 \%$ de los casos pediátricos, generalmente refleja el estado inflamatorio generalizado. La hepatomegalia ocurre en el $40 \%$ al $50 \%$ de los pacientes y hasta en el 25\% tiene pruebas de función hepática anormales. Las pruebas de función hepática marcadamente elevadas pueden ser vistas en hepatitis lupoide. 5 Las alteraciones del perfil hepático por lo general son leves $(<2$ veces el límite superior de lo normal y estas son, principalmente, elevaciones de aminotransferasas y fosfatasa alcalina (FA). ${ }^{10,11}$ 
Se ha descrito una variedad de enfermedades infecciosas, inflamatorias y neoplásicas que pueden producir linfadenitis necrosante. Dentro de los estudios infecciosos llevados a cabo solo se comprobó Herpes virus.

Desde el punto de vista clínico, la enfermedad de Kickuchi-Fujimoto y el lupus eritematoso sistémico pueden compartir diversas manifestaciones clínicas, como linfadenopatías, fiebre inexplicada.

De las enfermedades inflamatorias, el LES ha sido la más frecuentemente asociada a la enfermedad de Kickuchi-Fujimoto (EK) y su diagnóstico diferencial representa un reto. La ausencia en este caso de otras manifestaciones clínicas características del LES, asociada a unos anticuerpos antinucleares negativos, hacían poco probable este diagnóstico inicialmente. Sin embargo, la relación entre estas dos enfermedades es compleja y se debate si la enfermedad de Kickuchi-Fujimoto es una manifestación atípica del lupus eritematoso sistémico. ${ }^{6,7}$ En este caso se confirmó la linfadenitis necrotizante histiocítica por los hallazgos histopatológicos de predominio de CD8, CD68, CD20, infiltrado linfohistiocitario y cariorrexis sin neutrófilos. Algunos informes sugieren que el LES puede presentarse antes, al mismo tiempo o después de la resolución de los síntomas de la enfermedad de Kickuchi-Fujimoto. ${ }^{12.13}$

Por consiguiente, la EK y el Síndrome Hemofagocítico (SH) son enfermedades con características clínicas comunes que pueden asociarse entre sí y también con el LES, y pueden representar un importante desafío diagnóstico en determinados casos, llegando en ocasiones a superponerse a la enfermedad de base.

Muchos pacientes con EAI desarrollan desnutrición, que debe abordarse como parte del plan de tratamiento. En cuanto al tratamiento médico, la terapia de elección son los corticoides (budesonida y prednisona), ya que revierten el daño sistémico, revierte la pérdida auditiva súbita con sus efectos antiinflamatorios, inmunosupresores.

En muchos casos, el pronóstico dependerá del diagnóstico temprano y del tratamiento aplicado y, por ello, es fundamental su conocimiento en el contexto o no de una enfermedad infecciosa, autoinmune o neoplásica.

Este caso representó un reto diagnóstico por el espectro clínico y la evolución. Nuestro paciente debutó con hipoacusia progresiva, síndrome febril asociado a síntomas de malabsorción en presencia de un anticuerpo IgA anti-TGA positivo a títulos bajos, siendo probablemente un falso positivo, integrando sintomatología a un cuadro de enteropatía autoinmune. Nuestro paciente se le diagnosticó LES 1 año posterior a recaída luego de descenso de corticoides y estando con una estricta dieta libre de gluten.
Tabla 3. Manifestaciones clínicas en el lupus eritematoso sistémico (LES), la enfermedad de Kikuchi (EK) y el síndrome hematofagocítico $(\mathrm{SH}) .{ }^{12}$

\begin{tabular}{cccc}
\hline & LES & EK & SH \\
\hline Fiebre & ++ & + & +++ \\
Exantema & +++ & ++ & $+/-$ \\
Adenopatías & + & +++ & ++ \\
Citopenias & +++ & +++ & +++ \\
Artralgias & +++ & $+/-$ & $+/-$ \\
Encefalopatía & ++ & + & ++ \\
ANA & +++ & - & - \\
\hline
\end{tabular}

\section{Conclusiones}

Este caso demuestra la asociación con LES y manifestaciones gastrointestinales, en este caso enteropatía, hepatitis, síntomas de malabsorción. La atrofia de vellosidades tiene un diagnóstico diferencial amplio, no es sinónimo de enfermedad celíaca.

La pérdida auditiva súbita como debut de LES es inusual, mientras que la pérdida auditiva progresiva es más común; de hecho, puede estar subdiagnosticada. La hipoacusia súbita neurosensorial bilateral es reversible con el tratamiento esteroideo e inmunosupresor.

Otro aspecto importante que resaltar en este caso, es la necesidad de seguimiento de los pacientes a largo plazo, dada la compleja relación que existe entre el LES y otras enfermedades autoinmunes, y la presentación atípica en pacientes pediátricos. La decisión de tratar con una terapia más agresiva dependerá de la presencia de una entidad u otra y de las manifestaciones clínicas en cada caso

\section{Referencias}

1. Alves, SCB. Autoimmune gastrointestinal complications in patients with systemic lupus erythematosus: case series and literature review. Lupus (2016) 0, 1-11. DOI: $10.1177 / 0961203316655210$

2. Husby S, Koletzko S, Korponay-Szabó I, Kurppa K, Mea-rin ML, Ribes-Koninckx C, et al. European Society PaediatricGastroenterology, Hepatology and Nutrition Guidelines for Diag-nosing Coeliac Disease 2020. J Pediatr Gastroenterol Nutr.2020;70:141---56

3. Ferrara F, Quaglia S, Caputo I, Esposito C, Lepretti M, Pas-tore $S$, et al. Anti-transglutaminase antibodies in non-coeliacchildren suffering from infectious diseases. Clin Exp Immunol.2010;159:217---23.19.

4. Román Riechmann E et al. Aplicación racional de los nuevos criterios de la European Society for Paediatric Gastroenterology, Hepatology and Nutrition (ESPGHAN) 2020 para el diagnóstico de la enfermedad celíaca. An Pediatr (Barc). 2020;92(2):110.e1--110.e9

5. Rincón-Álvarez OJ, Neira-Torres, LI. Hearing loss and rheumatoid arthritis, systemic lupus erythematosus and Sjögren's syn- 
drome. Rev. Fac. Med. 2018 Vol. 66 No. 3: 439-46

6. Yehudai D, Shoenfeld Y, Toubi E. The autoimmune characteristics of progressive or sudden sensorineural hearing loss. AUTOIMMUNITY 2006; 39:153-158

7. Cramer JP, Schmiedel S, Alegre NG, Schäfer H, Burchard GD, Merz H. Necrotizing lymphadenitis: Kikuchi-Fujimoto disease alias lupus lymphadenitis? Lupus. 2010;19:89-92.

8. Mosquera JA, Romero S, Cruz J, Troncoso A, et al. Presentación simultánea de un caso de lupus eritematoso sistémico y enfermedad de Kikuchi. Su relación con el síndrome hematofagocítico y revisión de la bibliografía. Rev Esp Reumatol 2004;31(2):95-102

9. Benseler S, Silverman ED. Systemic Lupus Erythematosus Susanne M. Pediatr Clin N Am 52 (2005) 443 - 467
10. Betancur JF, Jimenez DF, Bonilla-Abadía F, Tobón GJ. Compromiso hepático de las enfermedades autoinmunes sistémicas. Rev colombreumatol. 2015;2 2(1):47-58.

11. Richer $\mathrm{O}$, Ulinski T, Lemelle I et al. Abdominal manifestations in childhood onset systemic lupus erythematosus. Ann Rheum Dis. 2007 Feb; 66(2): 174-178.

12. Gómez C, et al. Enfermedad de Kikuchi-Fujimoto: presentación de un caso pediátrico. Biomédica 2010;30:465-72

13. Mosquera JA, Romero S, Cruz J, Troncoso A, et al. Presentación simultánea de un caso de lupus eritematoso sistémico y enfermedad de Kikuchi. Su relación con el síndrome hematofagocítico y revisión de la bibliografía. Rev Esp Reumatol 2004;31(2):95-102 